\title{
Pesquisa epidemiológica descritiva: Estratégia pedagógica para o fortalecimento da análise de dados do Sistema Único de Saúde
}

\author{
Laís Ciribelli Yamaguchi, Rayane Evelin Brito Marques, Daniela Pimenta de Castro Fernandes,
} Jéssica Aline Silva Soares, Waneska Alexandra Alves

\begin{abstract}
Resumo
Atualmente, instituições de ensino superior (IES) têm reposicionado nos currículos disciplinas que apoiem o desenvolvimento de projetos de pesquisa em saúde, abrindo espaço ao método epidemiológico como instrumento importante para pesquisa em saúde pública. Oportuniza-se assim, ao discente de cursos da área da saúde o aprendizado das etapas de elaboração e as estratégias necessárias à pesquisa epidemiológica descritiva e analítica. Tendo como pressuposto que não há ciência sem pesquisa, incentiva-se o discente no pensar científico dos problemas em saúde segundo magnitude, relevância e pertinência regional. Portanto, justifica-se investigar o interesse temático em pesquisa dos estudantes, visto que influenciará diretamente suas práticas profissionais futuras. A disciplina "Epidemiologia" é oferecida aos estudantes de todos os cursos da área da saúde da Universidade Federal de Juiz de Fora, Campus Governador Valadares (UFJF-GV). Objetiva-se discorrer sobre o uso da pesquisa epidemiológica descritiva utilizando dados reais como recurso pedagógico no currículo dos cursos da área da saúde da UFJF-GV possibilitando assim o desenvolvimento do raciocínio crítico epidemiológico do discente. A disciplina tem como método avaliativo a apresentação de um boletim epidemiológico (BE), o qual se desenvolve em duas etapas, ao longo de um semestre letivo: inicia-se pela construção de um projeto de boletim, seguida da elaboração do BE propriamente dito. Para tanto, são escolhidas, livremente pelos alunos, doenças epidemiologicamente relevantes como objeto da pesquisa, dando ênfase às patologias de Minas Gerais registradas nos sistemas de informação do Sistema Único de Saúde (SUS) disponibilizada no sítio eletrônico do Departamento de Informática do SUS do Ministério da Saúde (Datasus/MS). Após coleta de dados sobre, principia-se a estruturação do projeto, o qual apresenta a proposta inicial do BE. O projeto é apresentado e discutido em seminário. Os dados são incrementados e analisados e calculados indicadores de morbimortalidade, a partir dos quais são construídas tabelas e gráficos, conforme atributos da epidemiologia descritiva e revisão de literatura científica. $\mathrm{O}$ resultado do estudo é apresentado por meio de trabalho escrito (BE) e seminário aberto. A disciplina possibilita o desenvolvimento do raciocínio crítico epidemiológico através da idealização e execução de projetos de análise de dados reais com temática aberta, fomentando a curiosidade e interesse dos acadêmicos para compreensão dos problemas de saúde local. Incentiva-se debate sobre aspectos relativos à fundamentação teórico-metodológica e técnicas da epidemiologia descritiva. Com o auxílio da pesquisa epidemiológica o discente atua como agente de transformação social, compreendendo o processo saúde-doença e as doenças/agravos relevantes localmente elaborando trabalhos científicos de alta credibilidade e valor acadêmico. A Epidemiologia contribui para a formulação, implementação e avaliação de políticas públicas de saúde. Os dados do Datasus/MS são importantes para a descentralização das ações de saúde, com foco na prevenção, controle e promoção de saúde. Os trabalhos e estudos desenvolvidos na disciplina conduziram os estudantes à leitura científica e ao pensamento epidemiológico baseado em evidência, possibilitando ao discente a viabilização de uma formação coerente com a política de saúde vigente, para uma melhor inserção dos futuros profissionais nos diversos cenários do SUS.
\end{abstract}

Descritores: Ensino em saúde, Epidemiologia, Análise de dados. 